\title{
The Motivating Role of Recovery Self-Disclosures From Therapists and Peers in Eating Disorder Recovery: Perspectives of Recovered Women.
}

\section{Citation}

Wasil, Akash, Katherine Venturo-Conerly, Rebecca Shingleton, and John Weisz. 2019. "The Motivating Role of Recovery Self-disclosures from Therapists and Peers in Eating Disorder Recovery: Perspectives of Recovered Women." Psychotherapy 56 (2): 170-180.

\section{Permanent link}

http://nrs.harvard.edu/urn-3:HUL.InstRepos:40827382

\section{Terms of Use}

This article was downloaded from Harvard University's DASH repository, and is made available under the terms and conditions applicable to Open Access Policy Articles, as set forth at http:// nrs.harvard.edu/urn-3:HUL.InstRepos:dash.current.terms-of-use\#OAP

\section{Share Your Story}

The Harvard community has made this article openly available. Please share how this access benefits you. Submit a story. 


\section{Psychotherapy}

\section{The Motivating Role of Recovery Self-Disclosures From Therapists and Peers in Eating Disorder Recovery: Perspectives of Recovered Women}

Akash Wasil, Katherine Venturo-Conerly, Rebecca Shingleton, and John Weisz Online First Publication, March 14, 2019. http://dx.doi.org/10.1037/pst0000214

CITATION

Wasil, A., Venturo-Conerly, K., Shingleton, R., \& Weisz, J. (2019, March 14). The Motivating Role of Recovery Self-Disclosures From Therapists and Peers in Eating Disorder Recovery: Perspectives of Recovered Women. Psychotherapy. Advance online publication.

http://dx.doi.org/10.1037/pst0000214 


\title{
The Motivating Role of Recovery Self-Disclosures From Therapists and Peers in Eating Disorder Recovery: Perspectives of Recovered Women
}

\author{
Akash Wasil, Katherine Venturo-Conerly, Rebecca Shingleton, and John Weisz \\ Harvard University
}

\begin{abstract}
Patients with eating disorders (EDs) often lack motivation to recover, and interventions designed to increase recovery motivation have not demonstrated to be effective. In fact, few studies have identified factors that increase recovery motivation in patients with EDs. We performed interviews with 13 women who recovered from EDs to identify factors that influenced their motivation to recover. Here, we present exploratory findings about a central theme from these interviews: the importance of hearing from others who had recovered from EDs (i.e., recovery self-disclosures [RSDs]). Of our 13 participants, 11 spontaneously reported that RSDs increased their motivation to recover. RSDs from therapists helped participants realize that recovery was possible, visualize the benefits of recovery, understand the recovery process, and develop stronger relationships with their therapists. RSDs from nontherapists produced similar benefits. Some of our participants who had become ED therapists after recovery reflected on the process of self-disclosing ED history to patients. They described when they choose to self-disclose (e.g., to boost patient motivation to change), what type of information they choose to self-disclose (e.g., information related to the patient's stage of recovery), and risks of recovery self-disclosures (e.g., stimulating patient competitiveness). Overall, our findings suggest that recovery self-disclosures may increase recovery motivation in patients with EDs. Limitations include discovering the theme of self-disclosure post hoc and exclusively interviewing participants who self-identified as recovered. Future research should identify if RSDs can be used in interventions to boost motivation for change and increase prorecovery behaviors, especially for patients with EDs.
\end{abstract}

\begin{abstract}
Clinical Impact Statement
Question: How do recovery stories from therapists and peers affect motivation to recover in patients with eating disorders (EDs)? Findings: In all, 11 of our 13 participants believed that hearing from recovered individuals improved their motivation to recover; therapists may encourage ED patients to speak with people who have recovered from an ED. Meaning: Hearing from recovered individuals may motivate ED patients by showing them that recovery is possible, teaching them about the recovery process, and strengthening the bond between patients and their therapists. Next Steps: Researchers should study interventions using recovery stories to see if they can improve motivation to recover in patients with EDs.
\end{abstract}

Keywords: motivation, eating disorders, recovery, self-disclosure, psychotherapy

Supplemental materials: http://dx.doi.org/10.1037/pst0000214.supp

I went to work at an eating recovery center. ... and nobody talked about the fact that they were in recovery. It was really hush hush. . . . it became kind of a struggle for me to determine: Do I tell people, or do I not? (Participant (P) 12; age: 35; formerly diagnosed with anorexia nervosa [AN] and eating disorder not otherwise specified [EDNOS])

Patients with eating disorders (EDs) often lack motivation to recover (Casasnovas et al., 2007). Of ED patients who do seek

Akash Wasil, Katherine Venturo-Conerly, Rebecca Shingleton, and John Weisz, Department of Psychology, Harvard University.

Correspondence concerning this article should be addressed to Akash Wasil, Department of Psychology, Harvard University, 344 Winthrop Mail Center, Cambridge, MA 02138. E-mail: akashwasil133@gmail.com treatment, many drop out prematurely (Mahon, 2000). For instance, among AN patients, between $20 \%$ and $51 \%$ drop out of inpatient treatment, and between $23 \%$ and $73 \%$ drop out of outpatient treatment (Fassino, Pierò, Tomba, \& Abbate-Daga, 2009). Furthermore, a meta-analysis of 99 randomized controlled trials concluded that about $25 \%$ of all ED patients drop out of treatment (Linardon, Hindle, \& Brennan, 2018). Given high dropout rates, interventions designed to decrease dropout and increase motivation may improve treatment outcomes.

Motivation to recover is a complicated phenomenon, and it is not fully clear if or how motivation to recover affects ED recovery. Studies vary in how they define and measure motivation (Vall \& Wade, 2015), and different types of motivation may relate differently to ED treatment outcomes. Some research suggests that cognitive/verbal measures of motivation are poor predictors of 
treatment outcomes, whereas behavioral measures of motivation may be more clinically useful (Waller, 2012). Nonetheless, metaanalytic data suggest that low motivation to recover contributes to high dropout rates and poor treatment outcomes (Vall \& Wade, 2015). Unfortunately, traditional motivation enhancement techniques (e.g., motivational interviewing and motivational enhancement therapy) appear to be ineffective for many ED patients (Knowles, Anokhina, \& Serpell, 2013; Waller, 2012). Because recovery motivation is associated with $\mathrm{ED}$ treatment outcomes and traditional motivational interventions are ineffective, studying new ways to motivate ED patients may be particularly valuable.

To our knowledge, no previous qualitative studies have focused exclusively or primarily on factors that motivate ED patients to recover. However, many studies have asked participants to identify factors that were helpful or harmful during their recovery process. In some of these studies, participants discussed factors that helped them recover. Examples include admitting to having a problem (D’Abundo \& Chally, 2004; Matusek \& Knudson, 2009; Pettersen \& Rosenvinge, 2002), seeing the dangers or negative consequences of their ED (Lamoureux \& Bottorff, 2005; Pettersen \& Rosenvinge, 2002), negative life events (D'Abundo \& Chally, 2004), feeling fed up (Pettersen \& Rosenvinge, 2002), involvement in meaningful causes (Matusek \& Knudson, 2009), and a desire to improve relationships (Linville, Brown, Sturm, \& McDougal, 2012).

Although past literature has identified some factors related to recovery motivation, further research is necessary to identify specific ways to instill or increase desire to recover in patients with EDs. That is, many factors identified by past studies may not be easy to target with interventions. For instance, it is not clear how therapists can help patients with EDs admit to having a problem or see the dangers of their EDs. Because little is known about factors that increase recovery motivation, research on this topic may help identify targets for future motivational interventions.

With this goal in mind, we conducted qualitative interviews with patients who were diagnosed with an ED and completed recovery (we refer to these individuals as "recovered ED patients") to identify factors that motivated them to recover. In this article, we present qualitative data on a powerful theme that emerged from the responses of our interviewees: the effect of recovery selfdisclosures (RSDs). We define the term "recovery selfdisclosures" as instances in which individuals who have recovered from an ED share their personal experiences with an ED. Notably, before the interviews, we did not expect participants to discuss the importance of RSDs.

RSD can be considered a specific form of self-disclosure. During self-disclosures, individuals (e.g., therapists or peers) share their own thoughts, feelings, and experiences with patients. A growing body of research suggests that self-disclosures improve therapeutic alliance (Audet \& Everall, 2010; Knox \& Hill, 2003) and possibly even treatment outcomes (Barrett \& Berman, 2001; Ziv-Beiman, Keinan, Livneh, Malone, \& Shahar, 2017) for patients with a variety of mental disorders. Specifically, selfdisclosures may improve therapeutic alliance by improving feelings of equality and comfort between patients and therapists (Knox, Hess, Petersen, \& Hill, 1997). In addition, strong therapeutic alliance has been associated with positive impressions of therapist self-disclosures (TSDs; Myers \& Hayes, 2006).
In the following sections, we review literature on TSDs and peer self-disclosures. Then, we explain how RSDs can be conceptualized as a specific form of self-disclosure. Lastly, we review the scarce research on RSDs and EDs.

\section{Therapist Self-Disclosures}

TSDs refer to instances in which therapists talk about their own feelings or past experiences (Audet \& Everall, 2010; Ziv-Beiman et al., 2017). TSDs have been discussed in relation to different psychotherapy approaches (Holmqvist, 2015; Knox \& Hill, 2003). For instance, early advocates of humanistic psychotherapies believed that TSDs strengthen the therapeutic alliance by conveying genuineness, empathy, and positive regard (Jourard, 1971; Rogers, 1961). In addition, although some psychodynamic theorists have recently promoted self-disclosures in therapy sessions, many psychodynamic therapists do not support the use of self-disclosures (Holmqvist, 2015). In feminist therapies, TSD is often viewed as a way to humanize the therapist and reduce power dynamics (Mahalik, Van Ormer, \& Simi, 2000). In cognitive-behavioral therapies, TSD is believed to help clients learn through modeling and reinforcement (Goldfried, Burckell, \& Eubanks-Carter, 2003). For instance, some cognitive-behavioral therapists disclose information about their training and disclose techniques that they use to address their own emotional and interpersonal issues (Holmqvist, 2015). Despite differing theoretical views on the use of selfdisclosures, self-disclosures may be common in clinical practice. One study found that $88.5 \%$ of therapy sessions in a university counseling center contained one or more TSD (Levitt et al., 2016).

In addition, researchers and therapists have debated the ethical consequences of TSDs in therapy (Audet, 2011). Although TSD may improve therapeutic alliance, normalize patient experiences, and improve patient perceptions of therapists, TSD can also break professional norms and violate boundaries in the therapeutic relationship (Audet, 2011). For instance, TSDs can cause the therapeutic relationship to feel like a friendship and cause clients to feel obligated to care for the therapist (Audet \& Everall, 2010; Henretty \& Levitt, 2010; Ziv-Beiman \& Shahar, 2016).

Therapists have mixed views about disclosing personal information to their patients. According to survey data, some believe that self-disclosure can be helpful in normalizing patient experiences, modeling helpful behaviors, providing new perspectives, and improving therapeutic alliance (Edwards \& Murdock, 1994; Simone, McCarthy, \& Skay, 1998). Other therapists believe that self-disclosure can remove attention from the patient, confuse the patient, and violate professional boundaries (Mathews, 1989; Simone et al., 1998). Notably, meta-analytic findings suggest clients share more information with therapists who self-disclose, view therapists who self-disclose more positively, and believe that therapists who self-disclose hold their clients in higher regard. (Henretty, Currier, Berman, \& Levitt, 2014).

The content in a self-disclosure may influence the effects of a self-disclosure (Levitt et al., 2016; Yeh \& Hayes, 2011). For instance, it is important to distinguish between TSDs that are intended to be therapeutic and TSDs that occur for other reasons. TSDs that are intentional, planned, and therapeutically motivated may have different effects than TSDs that are unintentional, spontaneous, or nontherapeutically motivated. In addition, empirical research has found TSDs with negative content have more favor- 
able effects than disclosures with positive content (Henretty et al., 2014), and therapists who disclose about resolved personal issues may be rated more favorably than therapists who disclose about ongoing personal issues (Yeh \& Hayes, 2011). Some kinds of self-disclosures have also been associated with specific kinds of outcomes. For instance, self-disclosures that reveal similarities between the therapist and client are associated with stronger therapeutic alliance and increased willingness to attend future sessions (Henretty et al., 2014). Moreover, TSDs intended to humanize the therapist are associated with postsession symptom improvements (Levitt et al., 2016).

To our knowledge, no studies have tested the effect of recovery therapist self-disclosures (RTSDs) on treatment outcomes, and only two studies have experimentally investigated the effects of TSDs. In one study, patients who were randomized to receive more TSDs experienced better treatment outcomes and liked their therapists more (Barrett \& Berman, 2001). In another study (ZivBeiman et al., 2017), patients with elevated symptoms on the Brief Symptom Inventory who were randomized to receive immediate TSDs (i.e., sharing information about therapists' in-session feelings or beliefs) experienced lower posttreatment symptoms than patients randomized to receive nonimmediate TSDs (i.e., sharing information about the therapist's life outside of therapy) or no TSDs (see Ziv-Beiman et al., 2017 for more information about immediate TSDs, nonimmediate TSDs, and other ways to classify TSDs). Notably, neither of these studies recruited patients with EDs.

\section{Peer Self-Disclosures and Peer Mentors}

Although little research has examined the role of RSDs from nontherapists (which we refer to as "recovery peer self-disclosures, or RPSDs"), research has demonstrated that peer support and peer mentor programs can be effective (Repper \& Carter, 2011). Some peer support programs involve structured interactions between peer support workers (i.e., individuals who have experienced a disorder) and patients with various mental illnesses (Repper \& Carter, 2011). Interactions between recovered individuals and current patients may provide patients with social support, coping skills, confidence, empowerment, hope for the future, and a variety of other benefits (for a review, see Repper \& Carter, 2011).

Given the benefits associated with peer mentorship, research on RPSDs may be especially valuable. RPSDs may confer some of the same benefits as peer mentorship (e.g., increased confidence and hope for the future). In addition, RPSDs occur often on online ED support forums (Kendal, Kirk, Elvey, Catchpole, \& Pryjmachuk, 2017) and may also occur in-person; research on the effects of RPSDs may provide useful recommendations to enhance the effects of peer support. Peer self-disclosures are also common in treatments for substance use disorders (Myrick \& del Vecchio, 2016). For patients with substance use disorders, peer support can improve access to treatment, increase engagement during treatment, and reduce symptoms (Myrick \& del Vecchio, 2016). Most relevant to this study on RSDs, RPSDs in substance use treatments have been shown to instill hope that it is possible to recover (Davidson, Bellamy, Guy, \& Miller, 2012). Substance use disorders and EDs share some key similarities (Wolfe \& Maisto, 2000), suggesting that patients with EDs may also benefit from RPSDs.
However, to our knowledge, no studies have examined the effects of RPSDs on patients with EDs. Even though studies have demonstrated that interactions with recovered individuals are helpful for patients with a variety of mental disorders (Repper \& Carter, 2011), these studies have not specifically examined the effects of RPSDs. In addition, because little past research on peer mentorship has involved patients with EDs (Repper \& Carter, 2011), it is unknown whether interactions with recovered individuals are helpful for patients with EDs.

\section{RTSDs and EDs}

Hearing from recovered individuals may be particularly motivating for patients with EDs, especially because they often believe that recovery is undesirable or unattainable. Because about $24 \%$ to $47 \%$ of ED therapists have past or current experience recovering from an ED (Barbarich, 2002; Warren, Schafer, Crowley, \& Olivardia, 2013), research examining TSDs with ED patients is especially relevant. Although past research on TSDs and peer selfdisclosures have rarely focused on patients with EDs, a few studies on self-disclosures have included patients with EDs. Some ED patients and ED therapists believe that experiential knowledge helps to enhance therapeutic alliance and promotes trust between patients and therapists (de Vos, Netten, \& Noordenbos, 2016; Johnston, Smethurst, \& Gowers, 2005). Despite these potential benefits, therapists rarely disclose past ED experiences to their patients. In one study of recovered ED therapists, only $12 \%$ disclosed their personal ED history (Warren et al., 2013). Some ED therapists reported that it is inappropriate to disclose to patients, and others feared that they would lose their job if employers discovered their ED history (Bloomgarden, Gerstein, \& Moss, 2003). In addition, although some ED programs have guidelines on the use of self-disclosure, many ED treatment centers lack guidelines for hiring or supervising therapists who have recovered from EDs (Costin \& Johnson, 2002).

Previous studies discussing the effect of TSDs on patients with EDs have recruited patients currently receiving therapy (de Vos et al., 2016) and therapists with a history of EDs (Warren et al., 2013). In addition to these perspectives, it may be valuable to hear from former patients who have recovered from their EDs. Recovered individuals may be able to offer different insights into the recovery process than patients currently receiving therapy. To our knowledge, no studies have examined the perspectives of recovered ED patients on TSDs. In addition, the one study that asked current ED patients about the effects of therapist disclosures recruited ED patients from a clinic in the Netherlands that exclusively hires therapists with personal ED history, trains them to use experiential knowledge, and advertises these aspects of its services (de Vos et al., 2016). Although the information gathered from this clinic is helpful, research on RSDs in more representative therapeutic settings (i.e., treatment centers that do not exclusively hire recovered ED therapists) may be more generalizable.

\section{RPSDs and EDs}

It may also be valuable to study the influence of peer selfdisclosures on patients with EDs. Therapists with and without personal ED histories can ask recovered individuals to speak with their patients. In addition, although no empirical research has 
assessed the impact of peer self-disclosures on patients with EDs, ED advocacy groups often encourage interactions between recovered individuals and current patients (Multi-Service Eating Disorders Association, 2018). Research on RSDs may help advocacy groups structure these interactions more effectively. In summary, no prior research has examined the effect of RPSDs on patients with EDs, and research on this topic could be useful for therapists, researchers, and advocacy groups.

\section{Current Study}

Despite the potential benefits of RTSDs and/or RPSDs, most standard treatment manuals for EDs do not address RSDs (Fairburn, 2008). Studying the effects of RSDs may yield valuable information for treatment outcome researchers designing interventions for patients with EDs, especially interventions designed to improve recovery motivation. Qualitative interviews with patients who have received RTSDs and RPSDs would likely improve our understanding of the effects of RSDs. Interviews highlight the lived experiences of patients, which may offer novel information to therapists and researchers. Themes from interviews can also generate hypotheses for further quantitative research.

In the current study, we conducted qualitative interviews with recovered ED patients to identify the factors that motivated them to recover. This article focuses on one of the major themes that emerged in these interviews: the role of RSDs from both peers and therapists. Our study expands on existing literature in several ways: (a) We present the views of recovered ED patients, (b) we present the views of patients who received therapy in typical therapeutic settings (i.e., therapeutic settings that do not necessarily have guidelines on self-disclosure), (c) we examine the role of both TSDs and peer self-disclosures, and (d) we focus on the role of disclosures in ED recovery motivation.

\section{Method}

\section{Participants}

A total of 13 participants were recruited from the Boston chapters of Project HEAL and the Multi-Service Eating Disorders Association via their e-mail lists and social media pages (see Table 1 for sample demographic characteristics and Figure S1 in online supplementary materials for CONSORT diagram). Based on sample size recommendations for qualitative studies (Crouch \& McKenzie, 2006; Guest, Bunce, \& Johnson, 2006), we aimed for a sample size of 12 to 15 participants. Past research suggests that qualitative studies reach saturation (i.e., a point at which no new information or themes are observed) within the first 12 participants (Guest et al., 2006). Participants eligible for this study were at least 18 years old, were previously diagnosed with an ED, received treatment for their ED, and recovered from their ED at least 1 year prior to the study. We limited recruitment to participants who had recovered for at least 1 year because past research shows that participants are less likely to relapse after a year (Khalsa, Portnoff, McCurdy-McKinnon, \& Feusner, 2017), and we aimed to identify motivating factors associated with long-term recovery. ED recovery was defined using two criteria: (a) Participants denied engaging in ED behavior including restriction, binge eating, and compensatory behaviors for at least 1 year; (b) all participants received
Table 1

Participant Demographics

\begin{tabular}{lc}
\hline \multicolumn{1}{c}{ Characteristic } & $M(S D)$ \\
\hline Age at interview & $33.09(7.19)$ \\
Age at disorder onset & $13.50(3.06)$ \\
Age at first ED diagnosis & $18.27(3.64)$ \\
Age of recovery & $29.00(5.50)$ \\
Mean EDE-Q total score & $0.68(.61)^{\mathrm{a}}$ \\
Median yearly household income & $\$ 75,000-\$ 99,000^{\mathrm{b}}$ \\
\hline & $N(\%)$ \\
\hline Gender & \\
Female & $11(100 \%)$ \\
Race & \\
White & $11(100 \%)$ \\
Highest education & $1(9 \%)$ \\
Associate degree & $2(18 \%)$ \\
Bachelor's degree & $8(73 \%)$ \\
Master's degree & \\
Most recent diagnosis & $4(36 \%)$ \\
Anorexia nervosa & $2(18 \%)$ \\
Bulimia nervosa & $5(45 \%)$ \\
EDNOS & $1(9 \%)$ \\
Most recent level of care & $5(45 \%)$ \\
Intensive outpatient & $4(36 \%)$ \\
Outpatient & $1(9 \%)$ \\
Residential &
\end{tabular}

Note. $\quad \mathrm{ED}=$ eating disorder; EDE-Q $=$ Eating Disorders Examination Questionnaire; EDNOS = eating disorder not otherwise specified.

${ }^{a}$ One participant had elevated scores on the Shape Concerns and Weight Concerns subscales of the EDE-Q but did not have an elevated total score. ${ }^{\mathrm{b}}$ Values represent median, not mean.

a total score on the Eating Disorders Examination Questionnaire (EDE-Q; Fairburn \& Beglin, 1994) within 1 SD of the community norm (Fairburn \& Beglin, 1994; Mond, Hay, Rodgers, \& Owen, 2006).

\section{Instruments}

Participants filled out a demographic survey and the EDE-Q (Fairburn \& Beglin, 1994). The EDE-Q is a self-report questionnaire widely used to assess ED attitudes and behaviors. It has acceptable internal consistency, test-retest reliability, and validity (Luce \& Crowther, 1999; Mond, Hay, Rodgers, Owen, \& Beumont, 2004). Specifically, internal consistency for each EDE-Q subscale was as follows (Luce \& Crowther, 1999): Restraint (Cronbach's $\alpha=.84$ ), Shape Concern (Cronbach's $\alpha=.93$ ), Weight Concern (Cronbach's $\alpha=.89$ ), and Eating Concern (Cronbach's $\alpha=.78$ ).

We developed a semistructured interview guide designed to identify the factors that influenced participants' decision to begin treatment and initiate their recovery process. To develop the interview guide, we reviewed past qualitative studies with ED patients (D'Abundo \& Chally, 2004; Matusek \& Knudson, 2009; Pettersen \& Rosenvinge, 2002), spoke with recovered individuals unaffiliated with the study, and brainstormed questions related to recovery motivation. All four authors reviewed and approved the final interview guide. Notably, none of our preprepared questions asked participants about the role of RSDs in their recovery process. 
Participants detailed the importance of self-disclosure in response to other questions, such as "What factors motivated you to seek treatment for your eating disorder?" and "Did anyone do anything that influenced your decision to recover? What was helpful, and what was harmful?".

\section{Procedure}

Prior to the semistructured interviews, participants completed a baseline survey including information on demographics, ED history, and the EDE-Q. Participants were randomly assigned to interview with the first author or the second author. All participants consented to participate in audio-recorded interviews. Data were de-identified; a spreadsheet linking participants' names to their transcripts was stored in a password-protected file. In addition, any identifying information was masked for publication purposes. We used Amazon Transcribe to transcribe the interviews. Members of the research team reviewed each of the transcripts produced by Amazon Transcribe for accuracy, and corrections were made as needed. Interviews were conducted in the winter and spring of 2018. Interviews lasted between 53 and $100 \mathrm{~min}(M=$ $75.00 \mathrm{~min}, S D=15.33 \mathrm{~min}$ ). Because the interviews addressed personal and emotional topics, participants were informed that they could terminate the interview at any time. After the interview, participants received a list of local resources (e.g., helplines), and participants had an opportunity to ask questions about the purpose of the study.

The four authors were the only members of the research team. The first two authors, undergraduate students studying psychology at Harvard University, conducted the interviews. They both have experience conducting research in several clinical psychology laboratories and clinical experience as peer counselors. In their role as peer counselors, licensed clinical psychologists trained them in nonjudgmental, nondirective counseling skills. The third author and fourth authors are licensed clinical psychologists trained in cognitive-behavioral techniques, and they both received a doctoral degree in clinical psychology. The third author has extensive experience conducting research on EDs, and the fourth author is an expert in youth and adolescent psychotherapy research. None of the authors have any conflicts of interest.

Before conducting the interviews, the first two authors completed a training involving mock interviews and discussions with the third author. From these discussions, the first two authors developed procedures to reduce bias, standardize interview procedures, and ensure fidelity to the semistructured interview guide. Although each interview varied based on the participant, the interviewers closely followed the guide in each interview. In addition, the first two authors debriefed after each interview to ensure that they were spending similar amounts of time on each question and asking similar follow-up questions.

\section{Analysis}

Verbatim transcripts were coded for key themes using thematic analysis (Braun \& Clarke, 2006). Initially, the first and second authors independently reviewed each of the transcripts to identify common themes (based on the number of participants who endorsed a particular theme). Then, discussions between the first two authors led to the development of a codebook detailing major topics from the interviews. Due to the high frequency of selfdisclosures in the interviews, the authors created a separate codebook centered around the theme of self-disclosures for the purposes of this article. We grouped comments related to selfdisclosure into categories based on whose perspective was being expressed (i.e., patient or therapist) and who delivered the RSD (i.e., therapist or peer). This led to the development of a codebook with three themes: (a) patient perspectives related to RTSD, (b) therapist perspectives related to RTSD (added to the codebook because some of our participants became therapists after recovery), and (c) patient perspectives related to non-RTSD. The first two authors independently assessed each of the full transcripts for the presence or absence of each of the themes in the codebook. For instance, if a participant mentioned the impact of a recovery self-disclosure from their therapist, we would code her transcript under the "patient perspectives related to RTSD" theme. Using Cohen's $\kappa$ coefficient, interrater reliability ranged from $k=0.86$ to $k=1.0$. After identifying the transcripts with these themes, the first two authors identified specific segments of each transcript that included information about self-disclosures. The authors then discussed ways to organize these segments into subthemes. Disagreements were resolved through discussion among the first three authors; all of the authors agreed on the final list of subthemes. The Harvard University Institutional Review Board approved the study.

\section{Results}

Of our 13 total participants, 11 spontaneously reported that hearing from recovered individuals affected their motivation to recover. See Table 1 for sample demographic characteristics of the 11 who are included in the analysis. All participants denied current ED behaviors and scored within community norms (Fairburn \& Beglin, 1994; Mond et al., 2006) for the EDE-Q total score (range: $0.0-1.18)$. Community norms for the EDE-Q total score $(M=$ $1.52, S D=1.25)$ were established in a large study of over 5,000 young women (Mond et al., 2006).

Based on the coded transcripts from these 11 participants, we identified three themes: (a) patient perspectives on RTSD, (b) therapist perspectives on RTSD, and (c) patient perspectives on peer (i.e., nontherapist) RSD (Table 2). In the following text, quotes from participants illustrate each of the themes within these three categories.

\section{RTSDs: Patient Perspectives}

Four participants reflected on their therapists revealing personal experience with EDs. All four participants found these instances of RTSD helpful, motivating, and inspiring. In particular, participants reported that recovered therapists provided evidence that recovery is possible, offered insight into the recovery process, identified false claims skillfully, and connected with patients using their personal experiences.

Providing evidence that recovery is possible. Four participants mentioned that conversations with recovered therapists helped them realize that people could recover from EDs. Conversations with recovered therapists provided hope and evidence that people could recover from EDs and live fulfilling lives. 
Table 2

Disclosure-Related Themes

\begin{tabular}{|c|c|c|}
\hline Theme & $\begin{array}{l}N \text { (percentage } \\
\text { of total sample) }\end{array}$ & Description/Sample quote \\
\hline $\begin{array}{l}\text { Recovery therapist self-disclosures: } \\
\text { Patient perspectives }\end{array}$ & $4(31 \%)$ & Participants reflected on hearing self-disclosures from their therapists \\
\hline $\begin{array}{l}\text { Providing evidence that recovery } \\
\text { is possible }\end{array}$ & $4(31 \%)$ & $\begin{array}{l}\text { [My therapist] was very, very up front with her own experience as a recovered therapist which, } \\
\text { looking back, I think that was really what did it for me ... My relationship with her, it } \\
\text { really changed my life. It was the first time I realized, oh, people can get better from this. } \\
\text { (P8, age: } 27 \text {; EDNOS) }\end{array}$ \\
\hline $\begin{array}{l}\text { Offering insight into the } \\
\text { recovery process }\end{array}$ & $4(31 \%)$ & $\begin{array}{l}\text { [My recovered therapists] taught me that recovery is definitely not a linear process.... . You're } \\
\text { gonna make mistakes. ... lapsing and relapsing is likely to happen. Just don't stay there. } \\
\text { (P6, age: } 41 \text {; AN and BN) }\end{array}$ \\
\hline $\begin{array}{l}\text { Connecting with therapists and } \\
\text { feeling understood }\end{array}$ & $3(23 \%)$ & $\begin{array}{l}\text { I felt like [my recovered therapist] could understand me because she had an eating disorder in } \\
\text { college. (P5, age: } 41 \text {; AN and EDNOS) }\end{array}$ \\
\hline $\begin{array}{l}\text { Recovery therapist self-disclosures: } \\
\text { Therapist perspectives }\end{array}$ & $4(31 \%)$ & Participants, who became therapists after recovery, reflected on disclosing to clients \\
\hline Choosing when to disclose & $3(23 \%)$ & $\begin{array}{l}\text { I think primarily when clients are really struggling with finding hope or reasons to get better, } \\
\text { or if a client is pretty stable in their process, then I might talk more in depth about my } \\
\text { experience with an eating disorder, like when I was at that stage in recovery kind of what } \\
\text { that looked like for me. (P12, age: } 35 \text {; AN and EDNOS) }\end{array}$ \\
\hline What to disclose & $4(31 \%)$ & $\begin{array}{l}\text { I think just being able to say, I totally get it, like I don't fully understand your experience } \\
\text { because we're different people, but I understand the struggle that you're sharing about fear } \\
\text { of weight gain, fear of letting go, not knowing who you are outside of the eating disorder, or } \\
\text { not having reasons to recover or not knowing reasons to recover. (P12, age: } 35 \text {; AN and } \\
\text { EDNOS) }\end{array}$ \\
\hline Perceive impact on patients & $4(31 \%)$ & $\begin{array}{l}\text { One to two times that I shared, it's been exceptionally effective. It's actually encouraged } \\
\text { clients to go [to higher levels of care]. They think, "oh well, my therapist went, and she } \\
\text { survived so I guess I can go!"” (P8, age: 27; prior diagnosis EDNOS) }\end{array}$ \\
\hline Recovery peer self-disclosures & $8(62 \%)$ & Participants reflected on recovery disclosures from nontherapists \\
\hline Proof that recovery is possible & $5(38 \%)$ & $\begin{array}{l}\text { I came into contact with recovered people, or people who were further along their recovery } \\
\text { path than me [through an advocacy group] . . . and I do feel that was really, really, really } \\
\text { important. . . . just like seeing a little bit of myself reflected in them and the fact that they } \\
\text { were okay, and they were saying they were happy-I didn't always believe them-but I } \\
\text { think it was really important for me to see that evidence over and over again. (P4, age: } 28 \text {; } \\
\text { AN) }\end{array}$ \\
\hline
\end{tabular}

Note. $\quad$ EDNOS $=$ eating disorder not otherwise specified; $\mathrm{AN}=$ anorexia nervosa; $\mathrm{BN}=$ bulimia nervosa.

[My therapist] was very, very up front with her own experience as a recovered therapist which, looking back, I think that was really what did it for me. . . My relationship with her, it really changed my life. It was the first Time I realized, oh, people can get better from this (P8, age: $27 ; \operatorname{EDNOS}^{1}$ )

One participant reported that it was particularly important to hear from recovered therapists toward the end of her recovery process. She explained that she would not have believed the stories of recovered individuals earlier in her recovery process.

I think it was important [toward the end of recovery]. . . . I think at the very beginning of my recovery if I had seen fully recovered people I would have probably been like uh like they're lying, or like that's not realistic. (P13, age 21; AN)

Offering insight into the recovery process. Four participants reported that hearing from recovered therapists allowed them to better understand the recovery process, including common challenges and misconceptions about recovery. For instance, one participant found it helpful when her recovered therapists explained how the recovery process often involves setbacks and mistakes.
[My recovered therapists] taught me that recovery is definitely not a linear process. . . . You're gonna make mistakes. . . . lapsing and relapsing is likely to happen. Just do not stay there. (P6, age: 41; AN and bulimia nervosa $[\mathrm{BN}])$

Hearing from recovered therapists also allowed participants to visualize their own recovery more realistically. For one participant, the ability to physically see the body of her recovered therapist was helpful. Many participants feared that recovery would bring misery and unstoppable weight gain; interacting with recovered people helped them combat that myth.

These were people that actually had been through it and had gone through gaining weight and weight restoration. I could look at them and go "oh ok, they look happy and healthy and they think it's not so bad to be a normal weight." (P5, age: 41; AN and EDNOS)

In addition, participants believed that therapists' stories helped participants understand recovery in ways that "seemed to make

\footnotetext{
${ }^{1}$ Diagnoses noted are past diagnoses, given sample was recovered.
} 
sense." Some therapists told patients personal anecdotes to help them understand and visualize recovery.

I remember my nutritionist giving me an example: She was like, 'I had an operation. ... I kind of felt sick to my stomach. The next day, I felt better, but I was really hungry. I was kind of munching all day long and rather than feel bad about that I was thinking, "gosh, you know, well it makes sense: My body needs more food because I really didn't eat that much yesterday, plus I'm recovering from an operation." She would give these little like, basic, gentle stories for me.... they talked to me in a way that recovery seemed to make sense. (P5, age: 41 ; AN and EDNOS)

Connecting with therapists and feeling understood. Three participants mentioned that they connected with their recovered therapists and felt understood due to their therapists' lived experience with an ED. One participant recounted an instance in which her recovered therapist related to her experience:

I was panicking about something pretty insignificant, and I was able to ask [my recovered therapist] "well, did you ever feel this way? . . And she would be like, "god, it's a completely real feeling-I experienced something like that." (P6, age: 41, AN and BN)

This participant also mentioned that her therapist was able to understand when hiding something or misrepresenting an experience. She believed that her recovered therapists drew from their personal experiences with EDs to identify and challenge harmful or disordered thoughts and behaviors.

"[Recovered therapists] had a better bullshit meter with that kind of stuff. I think part of that sometimes is because they probably, at one time in their life, had tried to do the same thing, and it's like yeah, "been there done that—not gonna work today, but thanks!" They had insight into things that unless you've been there, you do not necessarily pick up. There's only so much you can learn from a class-it's completely $100 \%$ different when you've lived it. (P6, age: 41; AN and $\mathrm{BN})$

Another participant felt like her recovered therapist was able to uniquely trust in her ability to recover because her therapist had recovered herself:

There was not anything in particular like that she did skill-wise or modality-wise, it was more just like, oh, this person cares about me. She has had my experience. She knows what it's like, but also she knows it gets better, and she trusts that I have the ability to get better. (P8, age: 27; EDNOS)

One participants felt like first-hand experience with an eating disorder allowed her therapists to better understand what she was experiencing. She said, "I felt like [my recovered therapist] could understand me because she had an eating disorder in college." (P5, age: 41 ; AN and EDNOS)

\section{RTSDs: Therapist Perspectives}

Four of our participants, who became ED therapists after recovery, reflected on their experiences disclosing their own ED recovery experiences to patients. Specifically, they reflected on choosing when they ought to disclose to their patients, the content they typically disclose, and the perceived effects of their selfdisclosures.
Choosing when to disclose. Three participants reflected on how they determined whether to disclose their experiences to their patients. One participant described this process as a "struggle," especially because the topic of TSD was rarely discussed at her treatment center.

I went to work at an eating recovery center. ... and nobody talked about the fact that they were in recovery. It was really hush hush. . . it became kind of a struggle for me to determine: Do I tell people, or do I not? (P12, age: 35; $\mathrm{AN}$ and EDNOS)

Although participants found it difficult to know when selfdisclosure would be helpful for a patient, some patient characteristics made therapists more likely to self-disclose. For instance, some participants were more likely to disclose to patients struggling to find motivation to recover.

I think primarily when clients are really struggling with finding hope or reasons to get better, or if a client is pretty stable in their process, then I might talk more in depth about my experience with an eating disorder, like when I was at that stage in recovery kind of what that looked like for me. (P12, age: 35; AN and EDNOS)

In addition, one participant was more likely to disclose to adolescents who questioned her authority as a therapist.

I worked pretty primarily with adolescents. I guess I am more encouraged to disclose if the client is more challenging, clients who say like, "well, what do you know?" That's not necessarily a window to be like, "oh, well, I know this." But I think younger clients do tend to bond more with people who understand or have empathy. True empathy. (P8, age: 27; EDNOS)

Participants also expressed concern that self-disclosure could harm some patients. One participant was less likely to disclose to participants who are especially competitive. She feared that competitive patients would make unhealthy comparisons between their own recovery process and that of their therapist. Another participant highlighted that some patients prefer to focus on their own concerns in therapy and that she is less likely to share her own experiences with these patients.

It really depends on my relationship with the client. I have some clients you absolutely do not want to- they're like, "oh yeah, thanks for sharing - but everything is about me." In those cases, I do not need to really bring up anything. It's like, ok, they know I'm in recovery, but I'm not going to try to use examples from my own recovery to try to help them. (P12, age: 35 ; AN and EDNOS)

What to disclose. Four participants reflected on the type of information they choose to disclose to clients. These therapists tended to self-disclose stories that related to the patient's current stage in the recovery process.

I think just being able to say, I totally get it, like I do not fully understand your experience because we're different people, but I understand the struggle that you're sharing about fear of weight gain, fear of letting go, not knowing who you are outside of the eating disorder, or not having reasons to recover or not knowing reasons to recover. (P12, age 35; AN and EDNOS)

Participants also emphasized that they could use self-disclosures to relate to patients' experiences. One participant mentioned that she 
would say, "I know how tough that is" (P9, age: 29; EDNOS) when she disclosed to her patients.

One participant disclosed her ED history to dispel the myth that recovery would cause excessive weight gain.

\begin{abstract}
This is probably unconventional, but I'm not overweight and I'm recovered. And so, sometimes I'll use that indirectly, to sort of validate the fact that this fear that we're going to continue to gain weight, gain weight, gain weight, really is not something that happens. ... Because I had that same fear, and that was not something that happened. (P12, age: 35; AN and EDNOS)
\end{abstract}

Perceived impact on patients. Four participants who were therapists believed that disclosing their ED history helped to motivate and support their patients. More specifically, these therapists believed that RSDs helped them support patients by improving trust and credibility.

[My patients] also told me that they can respect me a little bit more because I'm not just blowing smoke up their butt in that I know exactly what those crazy thoughts are. . . . sometimes they're more willing to share [crazy thoughts] with me. (P7, age: 45; AN)

I think peoples' ears perk up a little bit when they meet someone who, like, has been through the same experience. I see that in substance use treatment too, like they just take you a little bit more seriously. (P9, age: 29 ; EDNOS)

One participant reported that self-disclosure helped her persuade patients to seek higher levels of care:

One to two times that I shared, it's been exceptionally effective. It's actually encouraged clients to go [to higher levels of care]. They think, "oh well, my therapist went, and she survived so I guess I can go!" (P8, age: 27; prior diagnosis EDNOS)

\section{Recovery Peer Self-Disclosures}

Eight participants reported that hearing the stories of recovered peers who were not therapists could motivate recovery. Conversations with recovered peers often took place at ED advocacy meetings and support group meetings. In general, participants found these conversations helpful and motivating. One participant reflected, "The community_ having like a community of people that support your recovery or like people who have recovered themselves or are in recovery, is very, very motivating." (P13, age: 21; AN).

Five participants also believed that conversations with recovered peers could help them realize that ED recovery is possible. These interactions provided hope and motivation for participants' own recovery.

\footnotetext{
I came into contact with recovered people, or people who were further along their recovery path than me [through an advocacy group] . . . and I do feel that was really, really, really important. . . . just like seeing a little bit of myself reflected in them and the fact that they were okay, and they were saying they were happy-I didn't always believe them-but I think it was really important for me to see that evidence over and over again. (P4, age: 28 ; $\mathrm{AN})$
}

Some participants wished that they had spoken with a recovered peer. One believed that hearing from recovered people would have helped her understand the benefits of recovery.
To increase my motivation at the time I think that I needed to hear more about why recovery was worth it because I heard that a lot, like recovery's worth it, recovery's worth it, the way is recovery, but I never heard enough about why it was worth it. (P13, age: 21; AN)

Another believed that it would have been especially helpful to hear from a nontherapist:

I had never met someone who had identified themselves as having an eating disorder, so I never had that experience. Maybe if someone had said to me like "look, I've been through this and it gets better" other than the person who was treating me and who we were paying, maybe things would have been different. (P8, age: 27; EDNOS)

\section{Discussion}

The women in our study provided rich qualitative information about the perceived effects of hearing from recovered individuals; although these findings are exploratory, they may offer useful insights. Participants in our study reported that RSDs were motivating, inspiring, and helpful for their own recovery motivation. Overall, our study supports the idea that "therapists who have successfully mastered recovery become a living, breathing example that recovery is attainable" (Costin \& Johnson, 2002, p. 297), extends this idea to patients with EDs, and suggests that RPSDs may have similar effects. Specifically, patients believed that conversations with recovered peers and therapists made recovery seem more possible, more desirable, and more realistic. These data suggest a potential means to help individuals with EDs, increase motivation to seek treatment, and engage in recovery-oriented behaviors. Our participants also believed that recovered therapists could use their experiences to relate to participants and call out untrue statements. Therapists in our study believed that RSDs could help to motivate their patients, especially when disclosing to patients who struggled to find motivation to recover. Our results suggest that both RTSDs and RPSDs may increase recovery motivation for patients with EDs - raising an important hypothesis for future research.

Our findings, while exploratory, offer implications that can inform psychotherapy interventions for patients with EDs. Most notably, our study suggests that some patients with EDs find RSDs motivating, suggesting that therapists may wish to consider using RTSDs or RPSDs in interventions. More specifically, our study offers some potentially useful perspectives of therapists regarding whether or not to self-disclose. Therapists in our study were more likely to self-disclose to clients who struggled to find motivation to change ("when clients are really struggling with finding hope or reasons to get better") or questioned the credibility of their therapist ("clients who say like, 'well, what do you know?"). They were less likely to disclose to clients who preferred to focus on their own concerns ("They're like, 'oh yeah, thanks for sharingbut everything is about me."') and clients who were especially competitive.

The therapists in our study offered other comments that may be useful to therapists who do choose to use RSDs in interventions. Some of these comments suggest that when self-disclosing, therapists may wish to emphasize that recovery is possible (e.g., highlighting the ways in which their lives have changed after recovery), offer personal anecdotes that shed light on the recovery process (e.g., highlighting that recovery is nonlinear), and relate to participants' experiences (e.g., disclosing information relevant to 
the client's current stage of recovery). Therapists may also benefit from reports of how RSDs have led to change: Participants in our study reported that RSDs helped them believe that recovery is possible ("It was the first time I realized, oh, people can get better from this"), visualize recovery realistically ("I could look at them and go, 'oh, ok, they look happy and healthy, and they think it's not so bad to be a normal weight"'), and feel more understood by their therapists ("[My therapist] could understand me because she had an eating disorder.") In addition, therapists in our study believed that self-disclosures promote respect ("[clients] can respect me a little bit more because I'm not just blowing smoke up their butt"), trust ("sometimes [clients] are more willing to share [crazy thoughts] with me"), and credibility ("[clients] just take you a little bit more seriously").

Finally, it may be helpful for therapists to consider incorporating RPSDs in interventions. RPSDs could offer many of the benefits associated with RTSDs (e.g., providing proof that recovery is possible and offering insight into the recovery process) while limiting some of the risks (e.g., violating boundaries and negatively affecting the therapeutic relationship). RPSDs could be incorporated into interventions in a variety of ways: Therapists could bring recovered individuals into a therapy session, therapists could encourage patients to interact with recovered individuals outside of therapy sessions, or therapists could recommend books and social media pages created by recovered individuals.

Our study also adds to existing scientific literature on selfdisclosure in a few unique ways. First, although other qualitative studies have examined the effect of TSDs on patients in therapeutic settings (Audet \& Everall, 2003, 2010; Knox et al., 1997), our study is one of the first to focus on RSDs, a type self-disclosure in which therapists share their experience with a disorder. Our results suggest that RSDs may be a particularly valuable form of selfdisclosure for patients with EDs. Second, our study is the first to examine the role of RSDs on patients recovered from EDs. Although a previous study examined the effect of RSDs on patients actively receiving treatment for EDs (de Vos et al., 2016), the present study is the first to discuss the perspective of recovered ED patients. Although interviewing recovered ED patients limits our ability to draw conclusive statements about RSDs, because we do not know how often RSDs occur in patients who do not recover, there are also advantages of hearing from recovered ED patients. They may be well-equipped to reflect holistically on their recovery process and identify the most important factors that influenced their recovery. A recent review of 27 studies concluded that people who have been recovered for at least 1 year are less likely to relapse (Khalsa et al., 2017), suggesting that individuals who have recovered for at least 1 year may offer insight into factors associated with long-term recovery.

Third, our study includes participants' perspectives on both TSDs and peer self-disclosures. In contrast, previous research has only investigated either TSD or the effect of peer support (without addressing peer self-disclosure). Our data suggest that recovery stories from both therapists and peers may motivate patients to take steps toward recovery. In our study, many of the benefits traditionally associated with TSDs (Audet \& Everall, 2010) were also associated with peer self-disclosures. More specifically, our data suggest that hearing from recovered peers allows participants to believe that recovery is possible, to better understand the recovery process, and to perceive the benefits of recovery more clearly. RPSDs may be disseminated widely, as ED therapists with or without past ED experience could encourage patients to hear the stories of other recovered individuals. As noted earlier, peer selfdisclosures may also offer some of the benefits associated with TSD while avoiding some of the potential harms. For ethical reasons, therapists and researchers should obtain informed consent before organizing a RPSD, as RPSDs could involve sharing private health information. Fourth, our study identified recovery disclosures as a spontaneously generated theme. Given the strong influence of demand characteristics (Nichols \& Maner, 2008), it is possible that participants respond differently to interviews or questionnaires specifically designed to assess the role of therapist disclosure. Although our study does not fully eliminate the potential impact of demand characteristics, our study reduces their impact because our semistructured interview was not designed to ask participants about RSDs.

Our study has a few limitations, and our findings should be considered exploratory rather than conclusive. First, because our study used qualitative methods, our findings cannot be used to establish causal claims. Second, participants in our study were recruited at least 1 year after they had recovered from an ED. It is possible that participants who are currently receiving therapy would report differently about RSDs. Third, all of our participants were White women. Although this is common among research in EDs, it limits the generalizability of our findings. RSDs might differ in nature and impact across different racial and gender groups. For instance, participants might find RSDs more relatable and more relevant if the recovered person shares their racial or gender identity. Future research could involve interviews with men, racial minority groups, and nonrecovered individuals to better understand the effects of RSDs on these groups. Fourth, as our interview was not designed for therapists, we did not collect detailed information about the therapists in our study (e.g., theoretical orientation and years in practice) that may have added to our results. Fifth, the researchers who designed the study and conducted the interviews also transcribed and coded the transcripts. Although this is relatively common in qualitative ED research (de Vos et al., 2016; Pettersen \& Rosenvinge, 2002), it may have introduced bias into the study. Finally, all of our participants had recovered from their ED. It is possible that our sample underrepresented some of the harms associated with RSDs, or the number of patients for whom RSDs had no impact, as all of our participants ultimately recovered from their EDs.

Despite these limitations, our study suggests hypotheses and directions for future research. For instance, future research on selfdisclosures may wish to distinguish between RSDs and other types of self-disclosures; our findings suggest that RSD may be a specific type of disclosure that may offers unique motivational benefits. Importantly, past research has shown benefits associated with selfdisclosures that reveal negative content and establish similarities between therapists and clients (Henretty et al., 2014) — two characteristics of RSDs. Future studies could also test the effects of RSDs from both therapists and peers in treatment outcome studies and whether inclusions of RSDs enhance treatment outcome and increases prorecovery behavior change. In addition, future studies may identify patient (or therapist) characteristics that moderate the effect of RSDs. Finally, because different disorders benefit from different sources of motivation, future research may examine the effects of RSDs on specific EDs (e.g., patients with AN) and other mental disorders (e.g., 
patients with depression). A useful objective for the future will be clarifying, when, how, and for whom RSDs can improve desire to recover and potentially improve treatment outcomes.

\section{References}

Audet, C. T. (2011). Client perspectives of TSD: Violating boundaries or removing barriers? Counselling Psychology Quarterly, 24, 85-100. http://dx.doi.org/10.1080/09515070.2011.589602

Audet, C., \& Everall, R. D. (2003). Counsellor self-disclosure: Clientinformed implications for practice. Counselling and Psychotherapy Research, 3, 223-231. http://dx.doi.org/10.1080/14733140312331384392

Audet, C. T., \& Everall, R. D. (2010). TSD and the therapeutic relationship: A phenomenological study from the client perspective. British Journal of Guidance and Counselling, 38, 327-342. http://dx.doi.org/10 $.1080 / 03069885.2010 .482450$

Barbarich, N. C. (2002). Lifetime prevalence of eating disorders among professionals in the field. Eating Disorders, 10, 305-312. http://dx.doi .org/10.1080/10640260214505

Barrett, M. S., \& Berman, J. S. (2001). Is psychotherapy more effective when therapists disclose information about themselves? Journal of Consulting and Clinical Psychology, 69, 597-603. http://dx.doi.org/10 .1037/0022-006X.69.4.597

Bloomgarden, A., Gerstein, F., \& Moss, C. (2003). The last word: A "recovered enough" therapist. Eating Disorders, 11, 163-167. http://dx .doi.org/10.1080/10640260390199370

Braun, V., \& Clarke, V. (2006). Using thematic analysis in psychology. Qualitative Research in Psychology, 3, 77-101. http://dx.doi.org/10 $.1191 / 1478088706 q p 063$ oa

Casasnovas, C., Fernández-Aranda, F., Granero, R., Krug, I., JiménezMurcia, S., Bulik, C. M., \& Vallejo-Ruiloba, J. (2007). Motivation to change in eating disorders: Clinical and therapeutic implications. European Eating Disorders Review, 15, 449-456. http://dx.doi.org/10.1002/ erv.780

Costin, C., \& Johnson, C. L. (2002). Been there, done that: Clinicians' use of personal recovery in the treatment of eating disorders. Eating Disorders, 10, 293-303. http://dx.doi.org/10.1080/10640260214506

Crouch, M., \& McKenzie, H. (2006). The logic of small samples in interview-based qualitative research. Social Sciences Information Information Sur les Sciences Sociales, 45, 483-499. http://dx.doi.org/10 $.1177 / 0539018406069584$

D'Abundo, M., \& Chally, P. (2004). Struggling with recovery: Participant perspectives on battling an eating disorder. Qualitative Health Research, 14, 1094-1106. http://dx.doi.org/10.1177/1049732304267753

Davidson, L., Bellamy, C., Guy, K., \& Miller, R. (2012). Peer support among persons with severe mental illnesses: A review of evidence and experience. World Psychiatry, 11, 123-128. http://dx.doi.org/10.1016/j .wpsyc.2012.05.009

de Vos, J. A., Netten, C., \& Noordenbos, G. (2016). Recovered eating disorder therapists using their experiential knowledge in therapy: A qualitative examination of the therapists' and the patients' view. Eating Disorders, 24, 207-223. http://dx.doi.org/10.1080/10640266.2015 .1090869

Edwards, C. E., \& Murdock, N. L. (1994). Characteristics of TSD in the Counseling Process. Journal of Counseling and Development, 72, 384389. http://dx.doi.org/10.1002/j.1556-6676.1994.tb00954.x

Fairburn, C. G. (2008). Cognitive behavior therapy and eating disorders. New York, NY: Guilford Press.

Fairburn, C. G., \& Beglin, S. J. (1994). Assessment of eating disorders: Interview or self-report questionnaire? International Journal of Eating Disorders, 16, 363-370.

Fassino, S., Pierò, A., Tomba, E., \& Abbate-Daga, G. (2009). Factors associated with dropout from treatment for eating disorders: A compre- hensive literature review. BMC Psychiatry, 9, 67. http://dx.doi.org/10 .1186/1471-244X-9-67

Goldfried, M. R., Burckell, L. A., \& Eubanks-Carter, C. (2003). TSD in cognitive-behavior therapy. Journal of Clinical Psychology/In Session, 59, 555-568.

Guest, G., Bunce, A., \& Johnson, L. (2006). How many interviews are enough? An experiment with data saturation and variability. Field Methods, 18, 59-82. http://dx.doi.org/10.1177/1525822X05279903

Henretty, J. R., Currier, J. M., Berman, J. S., \& Levitt, H. M. (2014). The impact of counselor self-disclosure on clients: A meta-analytic review of experimental and quasi-experimental research. Journal of Counseling Psychology, 61, 191-207. http://dx.doi.org/10.1037/a0036189

Henretty, J. R., \& Levitt, H. M. (2010). The role of TSD in psychotherapy: A qualitative review. Clinical Psychology Review, 30, 63-77. http://dx .doi.org/10.1016/j.cpr.2009.09.004

Holmqvist, R. (2015). The use of self-disclosure among Swedish psychotherapists. European Journal of Psychotherapy and Counselling, 17, 80-98. http://dx.doi.org/10.1080/13642537.2014.996171

Johnston, C., Smethurst, N., \& Gowers, S. (2005). Should people with a history of an eating disorder work as eating disorder therapists? European Eating Disorders Review, 13, 301-310. http://dx.doi.org/10.1002/ erv. 659

Jourard, S. M. (1971). The transparent self (rev. ed.). Princeton, NJ: Van Nostrand.

Kendal, S., Kirk, S., Elvey, R., Catchpole, R., \& Pryjmachuk, S. (2017). How a moderated online discussion forum facilitates support for young people with eating disorders. Health Expectations, 20, 98-111. http:// dx.doi.org/10.1111/hex.12439

Khalsa, S. S., Portnoff, L. C., McCurdy-McKinnon, D., \& Feusner, J. D. (2017). What happens after treatment? A systematic review of relapse, remission, and recovery in anorexia nervosa. Journal of Eating Disorders, 5, 20. http://dx.doi.org/10.1186/s40337-017-0145-3

Knowles, L., Anokhina, A., \& Serpell, L. (2013). Motivational interventions in the eating disorders: What is the evidence? International Journal of Eating Disorders, 46, 97-107. http://dx.doi.org/10.1002/eat.22053

Knox, S., Hess, S. A., Petersen, D. A., \& Hill, C. E. (1997). A qualitative analysis of client perceptions of the effects of helpful TSD in long-term therapy. Journal of Counseling Psychology, 44, 274-283. http://dx.doi .org/10.1037/0022-0167.44.3.274

Knox, S., \& Hill, C. E. (2003). TSD: Research-based suggestions for practitioners. Journal of Clinical Psychology, 59, 529-539. http://dx.doi .org/10.1002/jclp. 10157

Lamoureux, M. M. H., \& Bottorff, J. L. (2005). "Becoming the real me": Recovering from anorexia nervosa. Health Care for Women International, 26, 170-188. http://dx.doi.org/10.1080/07399330590905602

Levitt, H. M., Minami, T., Greenspan, S. B., Puckett, J. A., Henretty, J. R., Reich, C. M., \& Berman, J. S. (2016). How TSD relates to alliance and outcomes: A naturalistic study. Counselling Psychology Quarterly, 29, 7-28. http://dx.doi.org/10.1080/09515070.2015.1090396

Linardon, J., Hindle, A., \& Brennan, L. (2018). Dropout from cognitivebehavioral therapy for eating disorders: A meta-analysis of randomized, controlled trials. International Journal of Eating Disorders, 51, 381391. http://dx.doi.org/10.1002/eat.22850

Linville, D., Brown, T., Sturm, K., \& McDougal, T. (2012). Eating disorders and social support: Perspectives of recovered individuals. Eating Disorders, 20, 216-231. http://dx.doi.org/10.1080/10640266.2012 .668480

Luce, K. H., \& Crowther, J. H. (1999). The reliability of the eating disorder examination-Self-report questionnaire version (EDE-Q). International Journal of Eating Disorders, 25, 349-351. http://dx.doi.org/10.1002/ (SICI)1098-108X(199904)25:3<349::AID-EAT15>3.0.CO;2-M

Mahalik, J. R., Van Ormer, E. A., \& Simi, N. L. (2000). Ethical issues in using self-disclosure in feminist therapy. In M. M. Brabeck (Ed.), Psychology of women book series. Practicing feminist ethics in psychol- 
ogy (pp. 189-201). Washington, DC: American Psychological Association. http://dx.doi.org/10.1037/10343-009

Mahon, J. (2000). Dropping out from psychological treatment for eating disorders: What are the issues? European Eating Disorders Review, 8, 198-216. http://dx.doi.org/10.1002/(SICI)1099-0968(200005)8: $3<198:: A I D-E R V 356>3.0 . C O ; 2-3$

Mathews, B. (1989). The use of TSD and its potential impact on the therapeutic process. Journal of Human Behavior and Learning, 6, 2529.

Matusek, J. A., \& Knudson, R. M. (2009). Rethinking recovery from eating disorders: Spiritual and political dimensions. Qualitative Health Research, 19, 697-707. http://dx.doi.org/10.1177/1049732309334077

Mond, J. M., Hay, P. J., Rodgers, B., \& Owen, C. (2006). Eating Disorder Examination Questionnaire (EDE-Q): Norms for young adult women. Behaviour Research and Therapy, 44, 53-62. http://dx.doi.org/10.1016/ j.brat.2004.12.003

Mond, J. M., Hay, P. J., Rodgers, B., Owen, C., \& Beumont, P. J. (2004). Validity of the Eating Disorder Examination Questionnaire (EDE-Q) in screening for eating disorders in community samples. Behaviour Research and Therapy, 42, 551-567. http://dx.doi.org/10.1016/S00057967(03)00161-X

Multi-Service Eating Disorders Association. (2018). Hope and inspiration. Retrieved from https://www.medainc.org/events/hope-inspiration/

Myers, D., \& Hayes, J. A. (2006). Effects of therapist general selfdisclosure and countertransference disclosure on ratings of the therapist and session. Psychotherapy, 43, 173-185. http://dx.doi.org/10.1037/00 33-3204.43.2.173

Myrick, K., \& Del Vecchio, P. (2016). Peer support services in the behavioral healthcare workforce: State of the field. Psychiatric Rehabilitation Journal, 39, 197-203. http://dx.doi.org/10.1037/prj0000188

Nichols, A. L., \& Maner, J. K. (2008). The good-subject effect: Investigating participant demand characteristics. Journal of General Psychology, 135, 151-166. http://dx.doi.org/10.3200/GENP.135.2.151-166

Pettersen, G., \& Rosenvinge, J. H. (2002). Improvement and recovery from eating disorders: A patient perspective. Eating Disorders, 10, 61-71. http://dx.doi.org/10.1002/erv.425

Repper, J., \& Carter, T. (2011). A review of the literature on peer support in mental health services. Journal of Mental Health, 20, 392-411. http://dx.doi.org/10.3109/09638237.2011.583947
Rogers, C. R. (1961). On becoming a person: A therapist's view of psychotherapy. Boston, MA: Houghton Mifflin Company.

Simone, D. H., McCarthy, P., \& Skay, C. L. (1998). An investigation of client and counselor variables that influence likelihood of counselor self-disclosure. Journal of Counseling and Development, 76, 174-182. http://dx.doi.org/10.1002/j.1556-6676.1998.tb02390.x

Vall, E., \& Wade, T. D. (2015). Predictors of treatment outcome in individuals with eating disorders: A systematic review and metaanalysis. International Journal of Eating Disorders, 48, 946-971. http:// dx.doi.org/10.1002/eat.22411

Waller, G. (2012). The myths of motivation: Time for a fresh look at some received wisdom in the eating disorders? International Journal of Eating Disorders, 45, 1-16. http://dx.doi.org/10.1002/eat.20900

Warren, C. S., Schafer, K. J., Crowley, M. E. J., \& Olivardia, R. (2013). Treatment providers with a personal history of eating pathology: A qualitative examination of common experiences. Eating Disorders, 21, 295-309. http://dx.doi.org/10.1080/10640266.2013.797318

Wolfe, W. L., \& Maisto, S. A. (2000). The relationship between eating disorders and substance use: Moving beyond co-prevalence research. Clinical Psychology Review, 20, 617-631. http://dx.doi.org/10.1016/ S0272-7358(99)00009-4

Yeh, Y.-J., \& Hayes, J. A. (2011). How does disclosing countertransference affect perceptions of the therapist and the session? Psychotherapy, 48, 322-329. http://dx.doi.org/10.1037/a0023134

Ziv-Beiman, S., Keinan, G., Livneh, E., Malone, P. S., \& Shahar, G. (2017). Immediate TSD bolsters the effect of brief integrative psychotherapy on psychiatric symptoms and the perceptions of therapists: A randomized clinical trial. Psychotherapy Research, 27, 558-570. http:// dx.doi.org/10.1080/10503307.2016.1138334

Ziv-Beiman, S., \& Shahar, G. (2016). Therapeutic self-disclosure in integrative psychotherapy: When is this a clinical error? Psychotherapy, 53, 273-277. http://dx.doi.org/10.1037/pst0000077

Received June 6, 2018

Revision received November 9, 2018

Accepted November 12, 2018 